\title{
Human acellular amniotic membrane incorporating exosomes from adipose-derived mesenchymal stem cells promotes diabetic wound healing
}

\section{Shune Xiao}

Department of Plastic Surgery, Nanfang Hospital, Southern Medical University

\section{Chunfang Xiao}

Department of Obstetrics and Gynecology, Nanfang Hospital, Southern Medical University

\section{Yong Miao}

Department of Plastic Surgery, Nanfang Hospital, Southern Medical University

\section{Jin Wang}

Department of Plastic Surgery, Nanfang Hospital, Southern medical University

\section{Ruosi Chen}

Department of Plastic Surgery, Nanfang Hospital, Southern Medical University

\section{Zhexiang Fan}

Department of Plastic Surgery, Nanfang Hospital, Southern Medical University

Zhiqi Hu ( $\nabla$ huzhiqidr163@i.smu.edu.cn )

Nanfang Hospital, Southern Medical University https://orcid.org/0000-0002-1657-0396

\section{Research}

Keywords: adipose-derived mesenchymal stem cells, exosomes, diabetic wound healing, acellular amniotic membrane

Posted Date: January 25th, 2021

DOl: https://doi.org/10.21203/rs.3.rs-151677/v1

License: (a) (1) This work is licensed under a Creative Commons Attribution 4.0 International License. Read Full License 


\section{Abstract}

Background: Diabetic wounds threaten the health and quality of life of patients and their treatment remains challenging. ADSC-derived exosomes have shown encouraging results in enhancing diabetic wound healing. However, the common method of exosome administration is subcutaneous injection at several sites around the wound, causing further damage and preventing direct contact between the exosomes and the injury site.

Methods: A diabetic mouse skin wound model was established. ADSC-derived exosomes (ADSC-Exos) were isolated and in vitro application of exosomes was evaluated using human umbilical vein endothelial cells (HUVECs) and human dermal fibroblasts (HDFs). After preparation and characterization of a scaffold of human acellular amniotic membrane (hAAM) loaded with ADSC-Exos in vitro, they were transplanted into wounds in vivo and wound healing phenomena were observed by histological and immunohistochemical analyses to identify the wound healing mechanism of the exosome-hAAM composites.

Results: The hAAM scaffold dressing was very suitable for the delivery of exosomes. ADSC-Exos enhanced the proliferation and migration of HDFs and promoted proliferation and tube formation of HUVECs in vitro . In vivo results from a diabetic skin wound model showed that the hAAM-Exos dressing accelerated wound healing by regulating inflammation, stimulating vascularization and promoting the production of extracellular matrix.

Conclusion: Exosome-incorporated hAAM scaffolds showed great potential in promoting diabetic skin wound healing, while also providing strong evidence for the future clinical applications of ADSC-derived exosomes.

\section{Full Text}

Due to technical limitations, full-text HTML conversion of this manuscript could not be completed. However, the manuscript can be downloaded and accessed as a PDF.

\section{Figures}



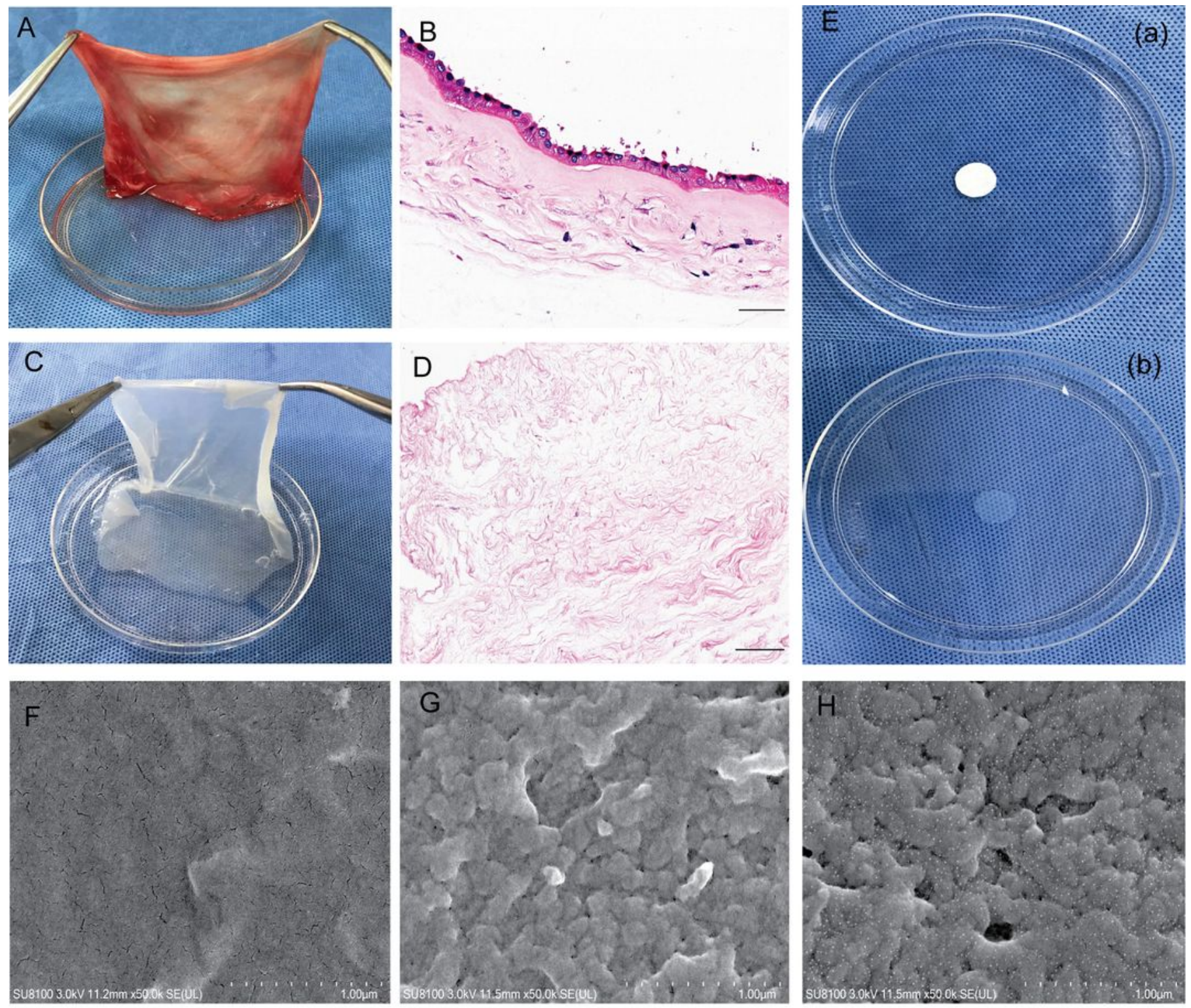

I

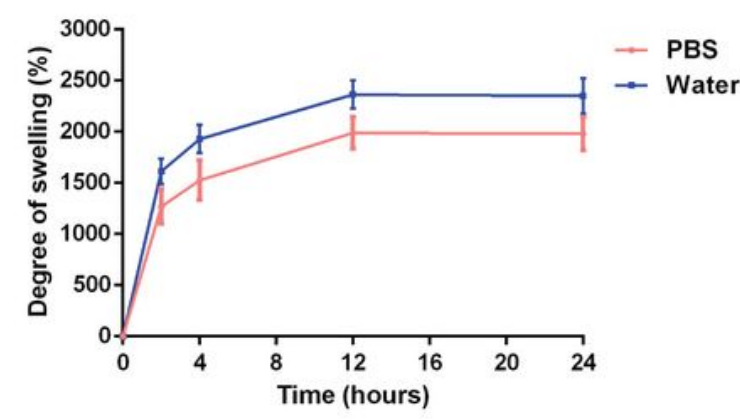

$J$

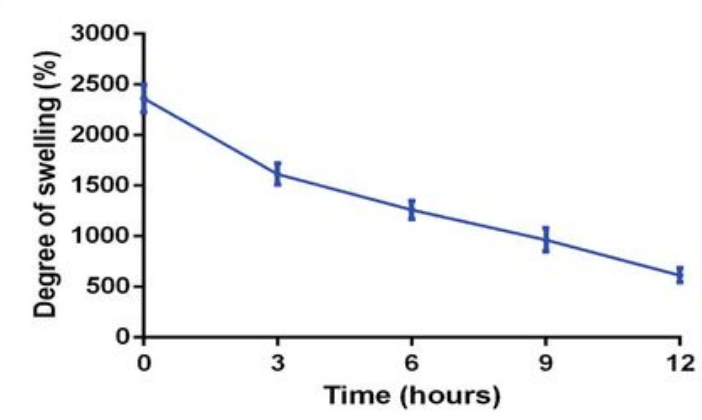

\section{Figure 1}

Characterization of human acellular amniotic membrane (hAAM). (A) The appearance of human amniotic membrane (hAM) tissue before decellularization. (B) Hematoxylin \& eosin (H\&E) staining of hAM before decellularization, showing the presence of a large number of nuclei (scale bar $=50 \mu \mathrm{m}$ ). (C) The appearance of hAM after decellularization when the tissue became transparent. (D) H\&E staining of hAM after decellularization showing almost no cell residue (scale bar $=50 \mu \mathrm{m}$ ). (E) Morphology of dry 
hAAM (a) and wet hAAM (b). (F) SEM images of the dry hAAM surface (scale bar $=1 \mu \mathrm{m})$. (G) SEM images of the wet hAAM surface (scale bar $=1 \mu \mathrm{m})$. $(\mathrm{H})$ Scanning electron microscopy images of the wet hAAM surface after adding exosomes (scale bar $=1 \mu \mathrm{m}$ ). (I) Swelling degree of the hAAM in different media at different time-points. $(\mathrm{J})$ Moisture retention capacity of the hAAM.

A

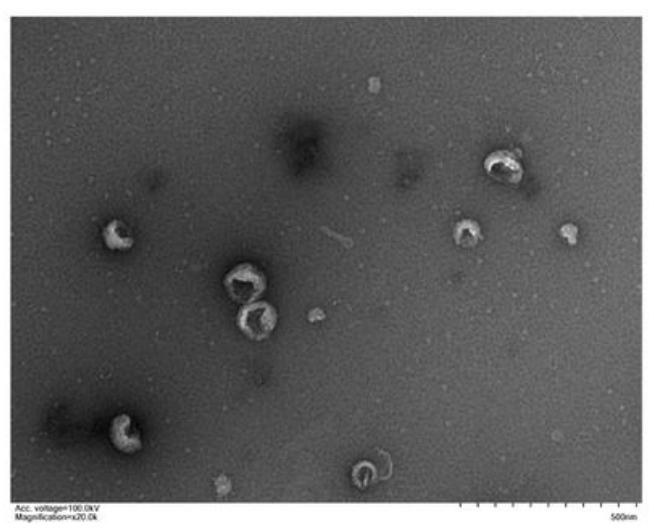

B

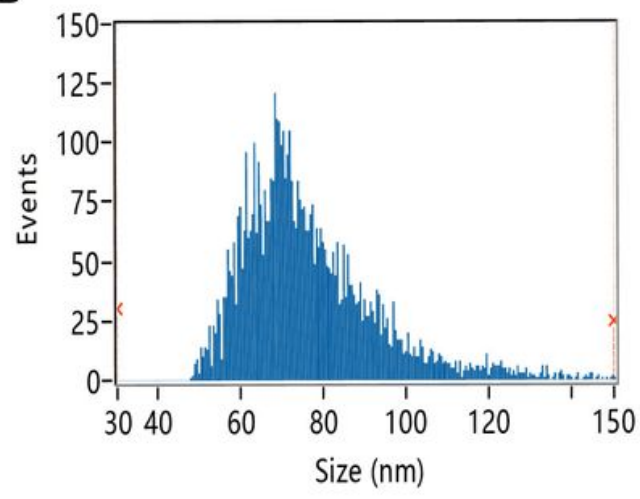

C

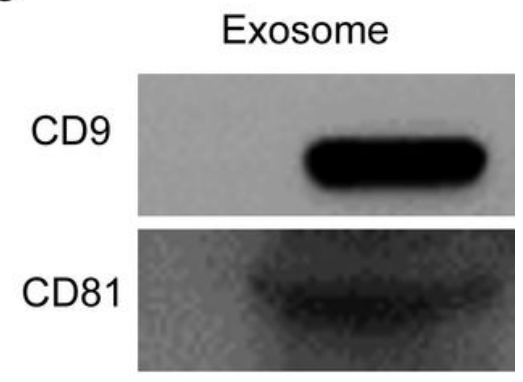

\section{Figure 2}

Characterization of ADSC-derived exosomes. (A) Representative images showing the morphology of ADSC-derived exosomes by transmission electron microscopy (scale bar $=500 \mathrm{~nm}$ ). (B) NTA analysis demonstrating the diameter of exosomes which ranged from 47.7 to $150.0 \mathrm{~nm}$, with a mean diameter of $76.4 \mathrm{~nm}$. (C) Expression of exosomal markers (CD9 and CD81) examined by western blot analysis. 
A

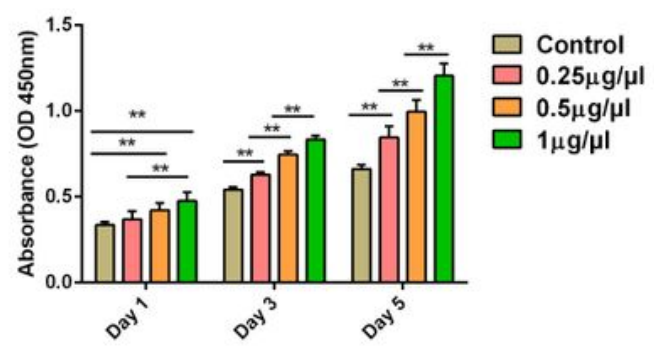

B

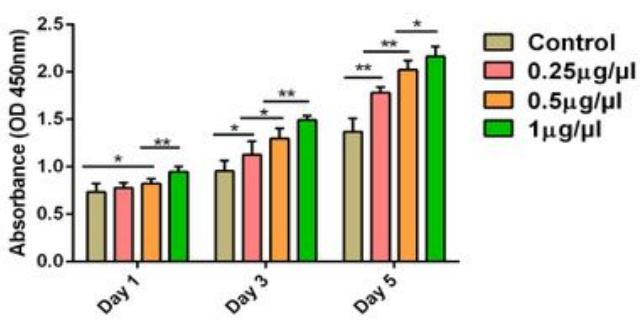

C

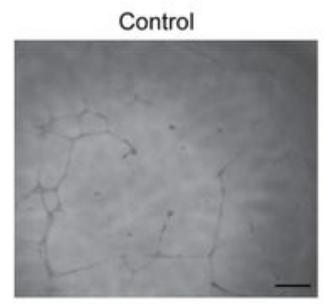

$0.5 \mu \mathrm{g} / \mu \mathrm{l}$

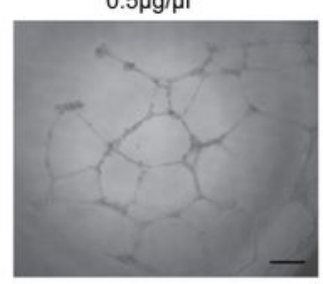

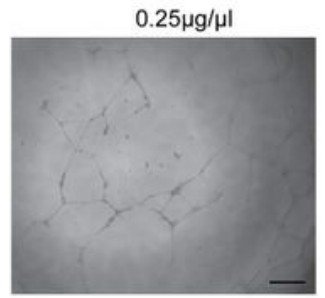

$1 \mu \mathrm{g} / \mu \mathrm{l}$

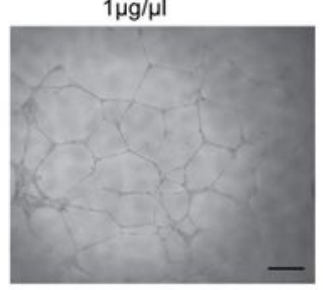

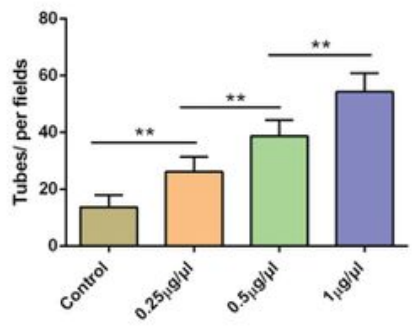

D

Control

$0.25 \mu \mathrm{g} / \mu \mathrm{l}$

$0.5 \mu \mathrm{g} / \mu \mathrm{l}$

$1 \mu \mathrm{g} / \mu \mathrm{l}$
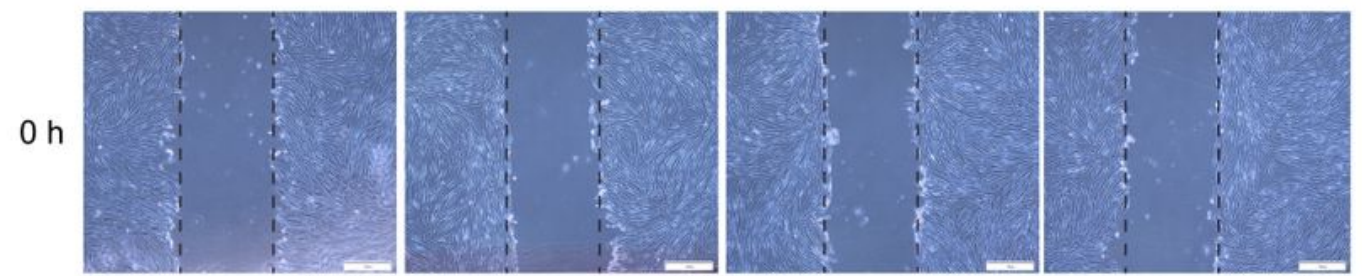

$12 \mathrm{~h}$
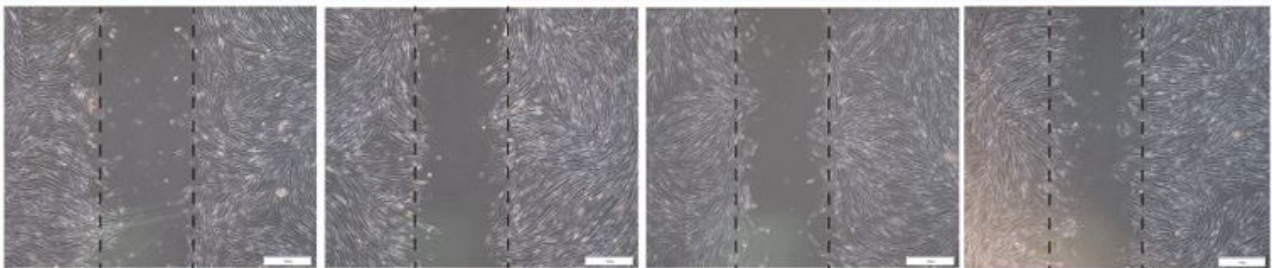

$24 \mathrm{~h}$
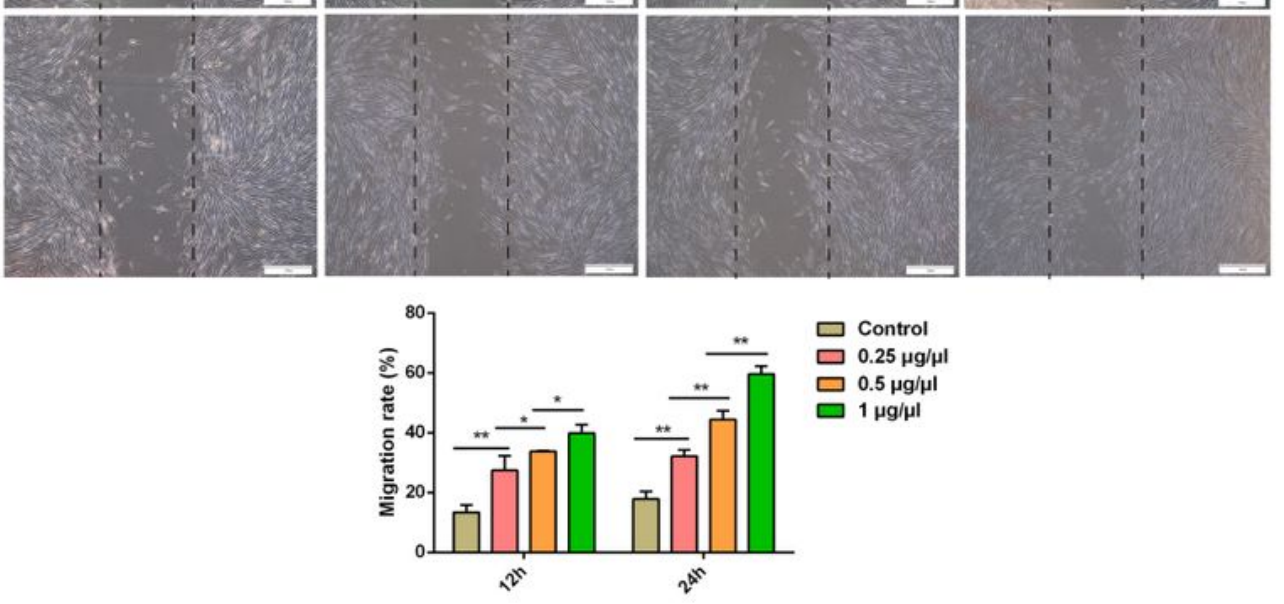

\section{Figure 3}

In vitro assessment of the effects of ADSC-derived exosomes on HUVECs and HDFs. Exosomes promoted the proliferation of HUVECs and HDFs in a dose-dependent manner. Exosomes promoted the tubeforming ability of HUVECs and the migration of HDFs also in a dose-dependent manner. (A) CCK-8 assay results of HUVECs. (B) CCK-8 assay results of HDFs. (C) Tube formation results of HUVECs after $24 \mathrm{~h}$. 
Scale bar $=500 \mu \mathrm{m}(\mathrm{D})$ Cell migration results of HDFs at 12 and $24 \mathrm{~h}$. Scale bar $=500 \mu \mathrm{m} .{ }^{\star \star P} \mathrm{P}<0.01,{ }^{\star} \mathrm{P}<$ 0.05 .

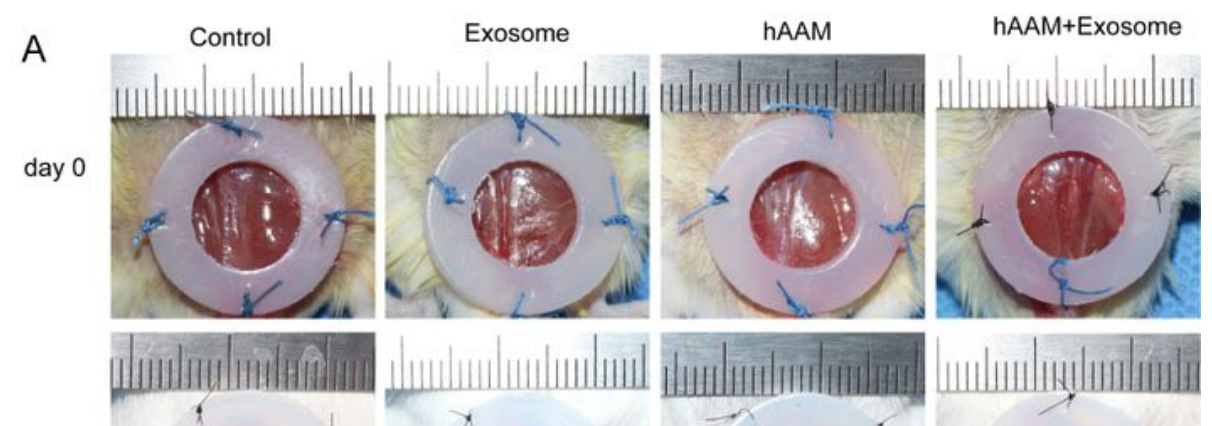

day 3

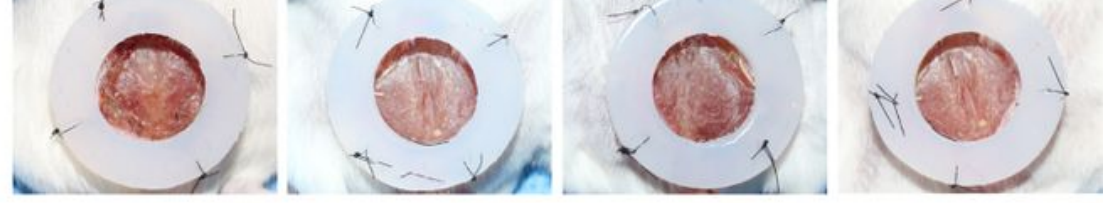

day 7
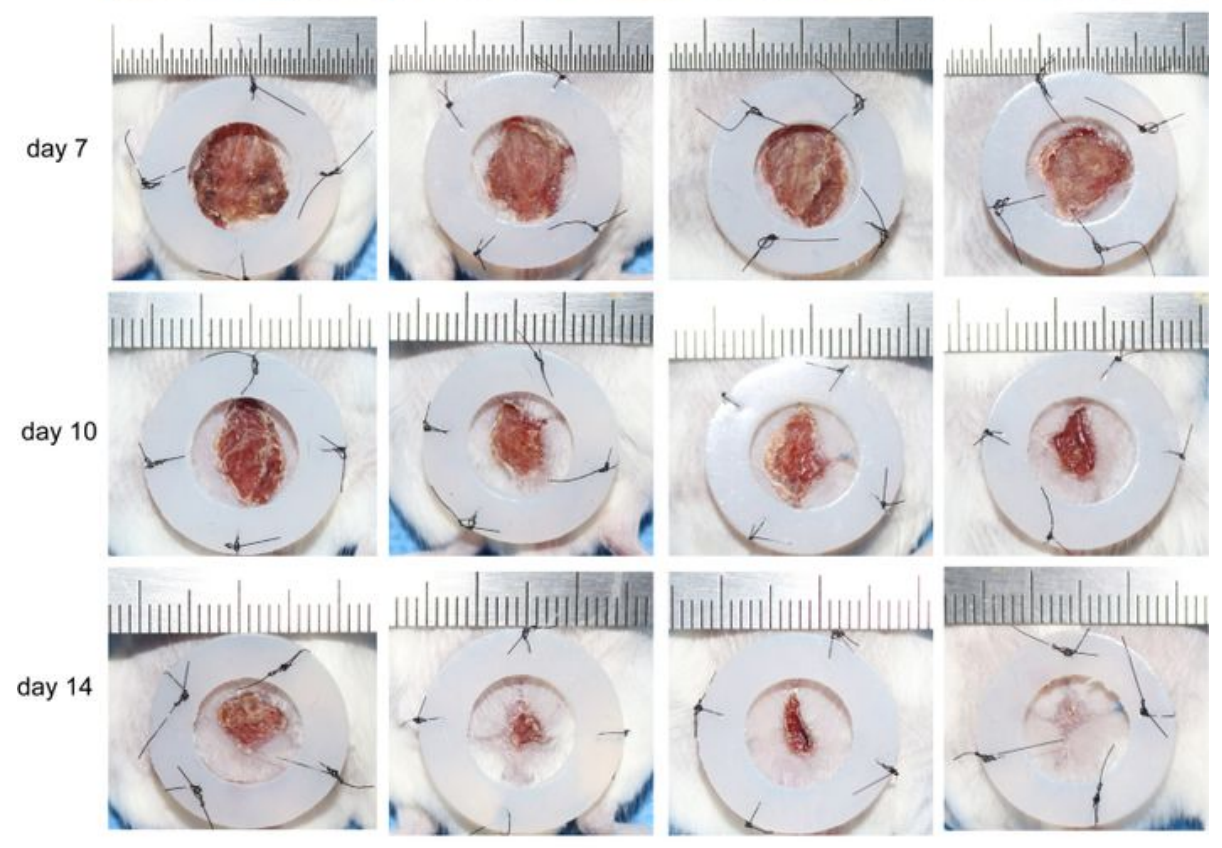

B

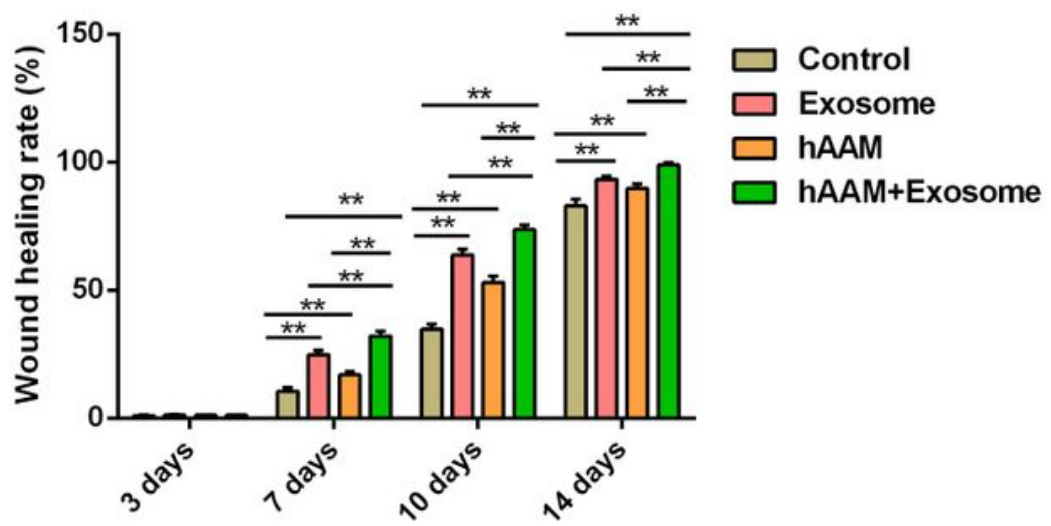

Figure 4

Healing process of diabetic wounds promoted by the hAAM-Exos scaffold dressing. (A) Representative images of the wound healing process in diabetic mice treated with control, hAAM, exosomes, or hAAMExos. (B) Wound closure rates at different time points of the four groups; $* * P<0.01, * P<0.05$. 


\section{A}
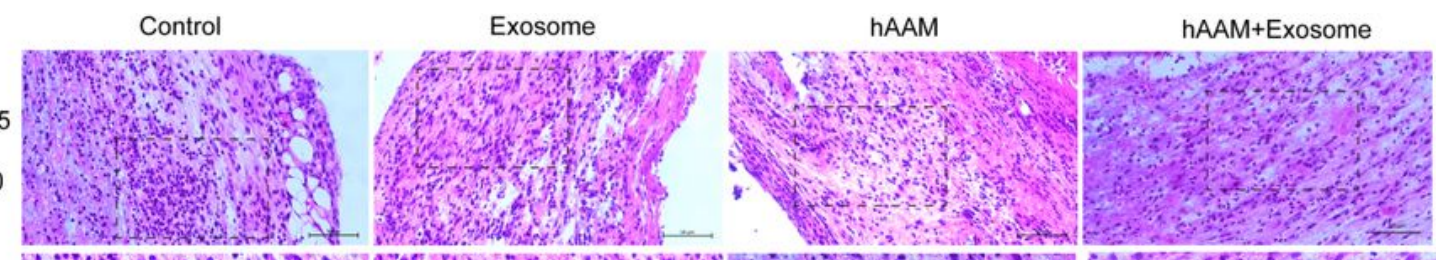

$\times 400$

Whas and

2.7.
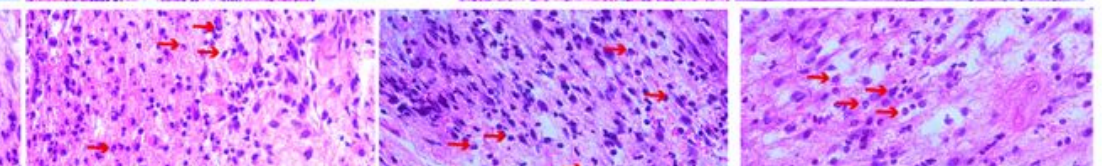

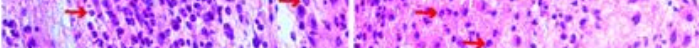
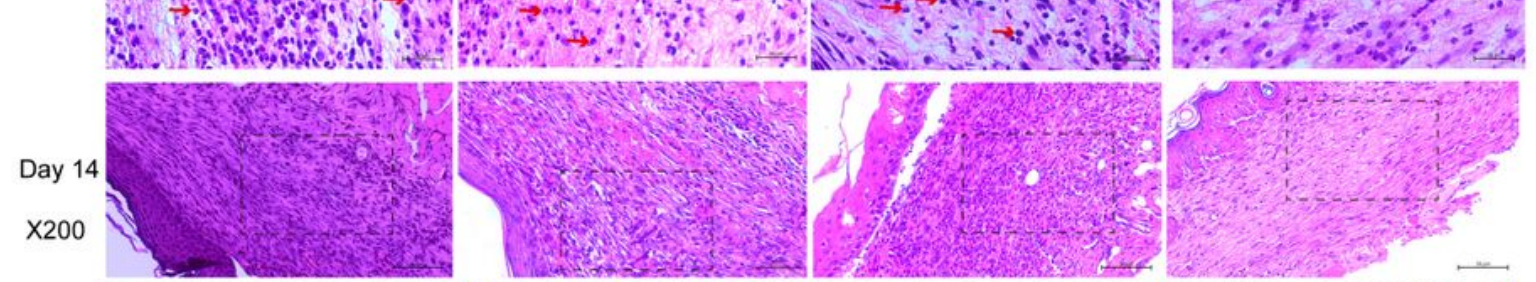

$\mathrm{x} 400$
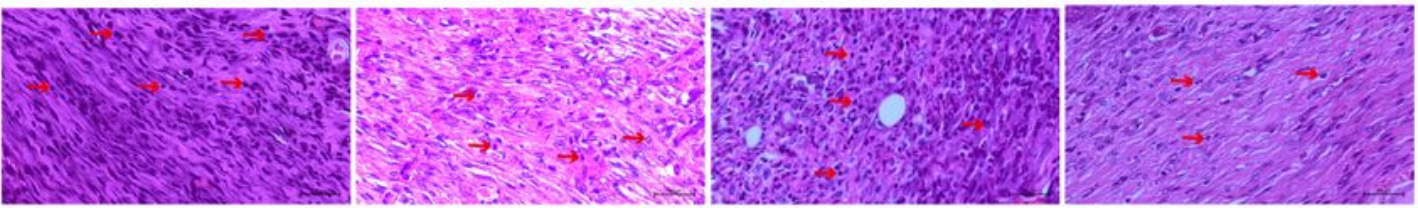

B

(a)

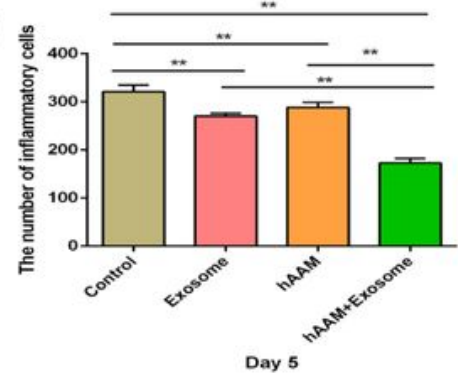

(b)

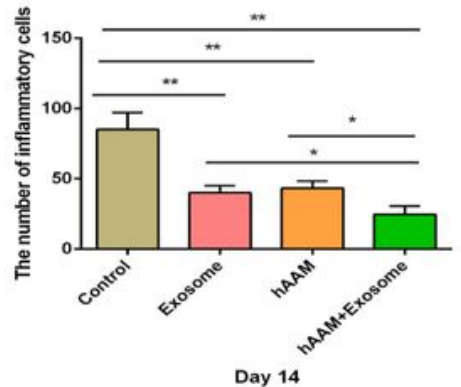

C

Control

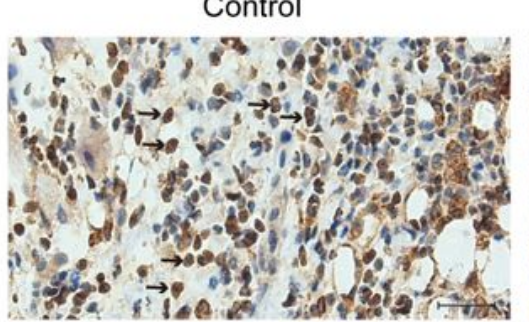
hAAM

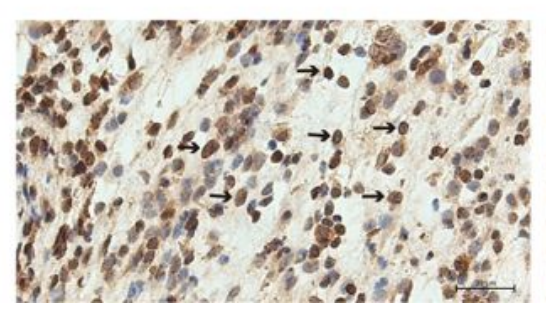

Exosome

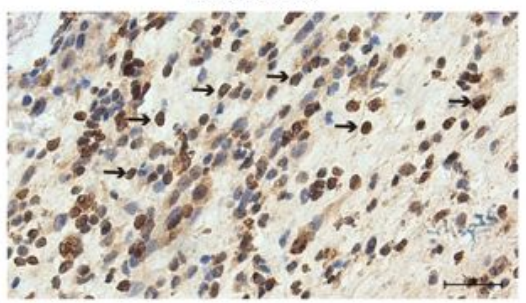

hAAM+Exosome
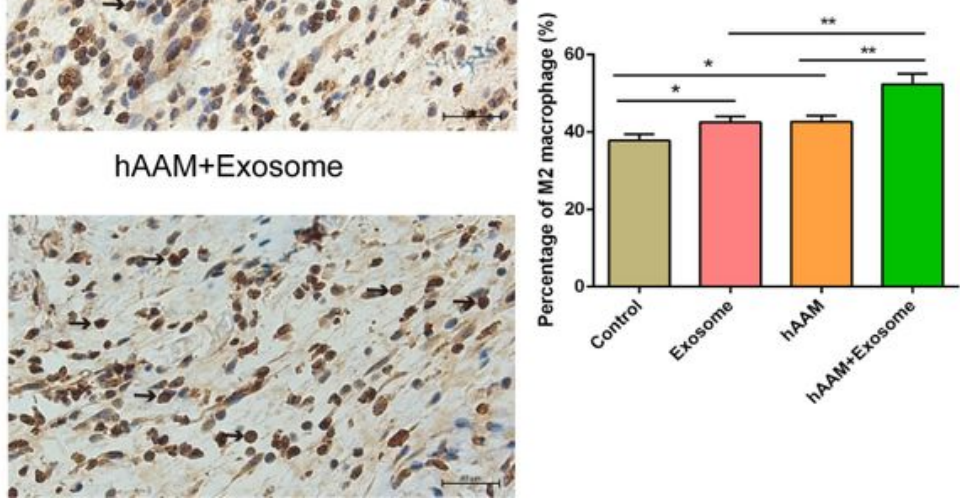

\section{Figure 5}

Infiltration of inflammatory cells during wound healing. The hAAM-Exos group showed the lowest number of inflammatory cells at days 5 and 14 and the highest proportion of anti-inflammatory cells (CD206+) at day 5. (A and B) Representative images and quantification results of inflammatory cells evaluated by H\&E staining in all groups at days 5 and 14. The 200x image scale bar $=50 \mu \mathrm{m}$; the 400x image scale bar 
$=20 \mu \mathrm{m}$. (C) Representative images and quantification results of CD206+ cells by immunohistochemical staining at day 5 . Scale bar $=20 \mu m ; * * P<0.01, * P<0.05$.

A Control
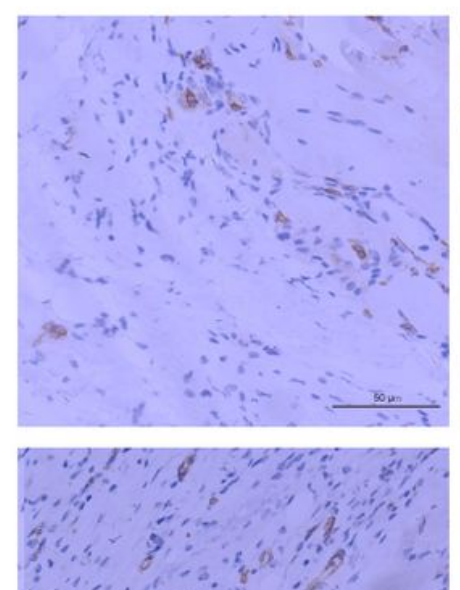

day 14

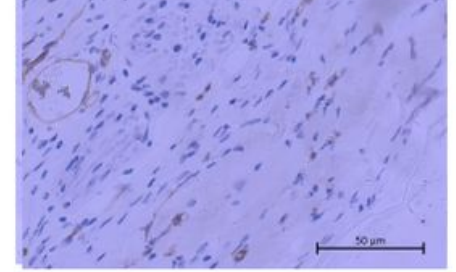

B
Exosome
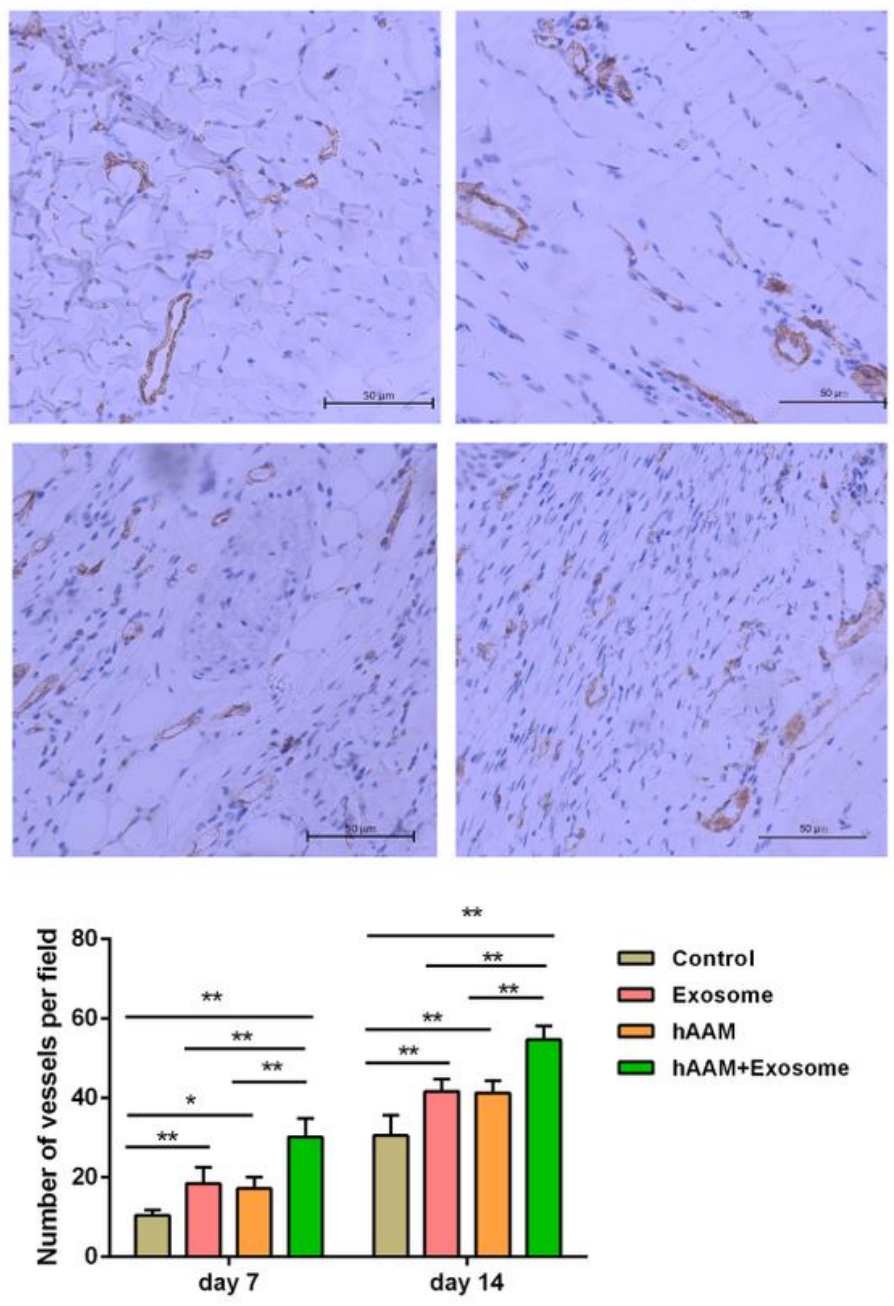

hAAM

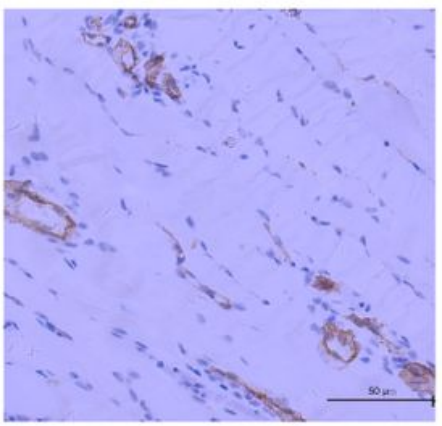

$\square$ Control

$\square$ Exosome

$\square$ hAAM

$\square$ hAAM+Exosome
hAAM+Exosome
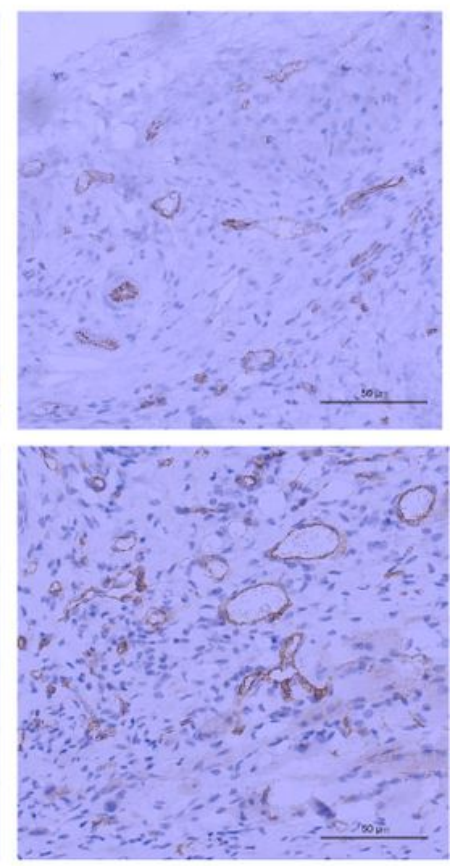

\section{Figure 6}

Immunohistochemical analysis of angiogenesis. The hAAM-Exos group showed the highest vessel numbers and densities. (A) Representative images of immunohistochemical staining of CD31 in each group at day 7 and day 14 . Scale bar $=50 \mu \mathrm{m}$. (B) Quantitative analysis of the number of vessels per field in each group at day 7 and day $14 .{ }^{* *} \mathrm{P}<0.01,{ }^{*} \mathrm{P}<0.05$. 
A

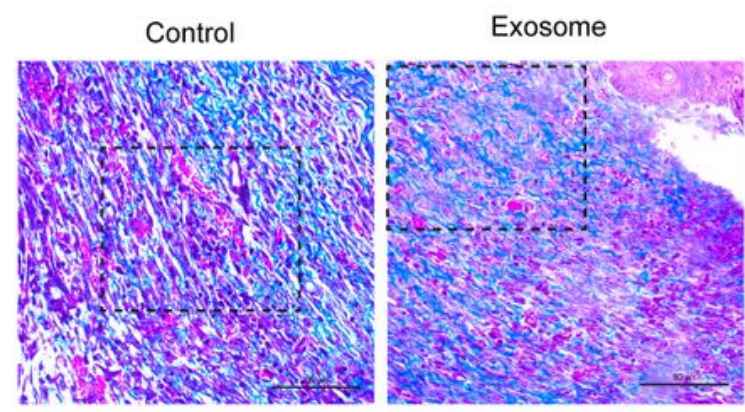

$\times 400$

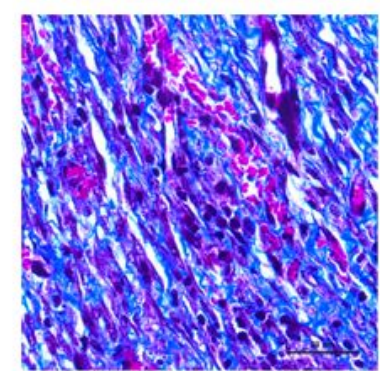

Day 14

X200

$\times 400$
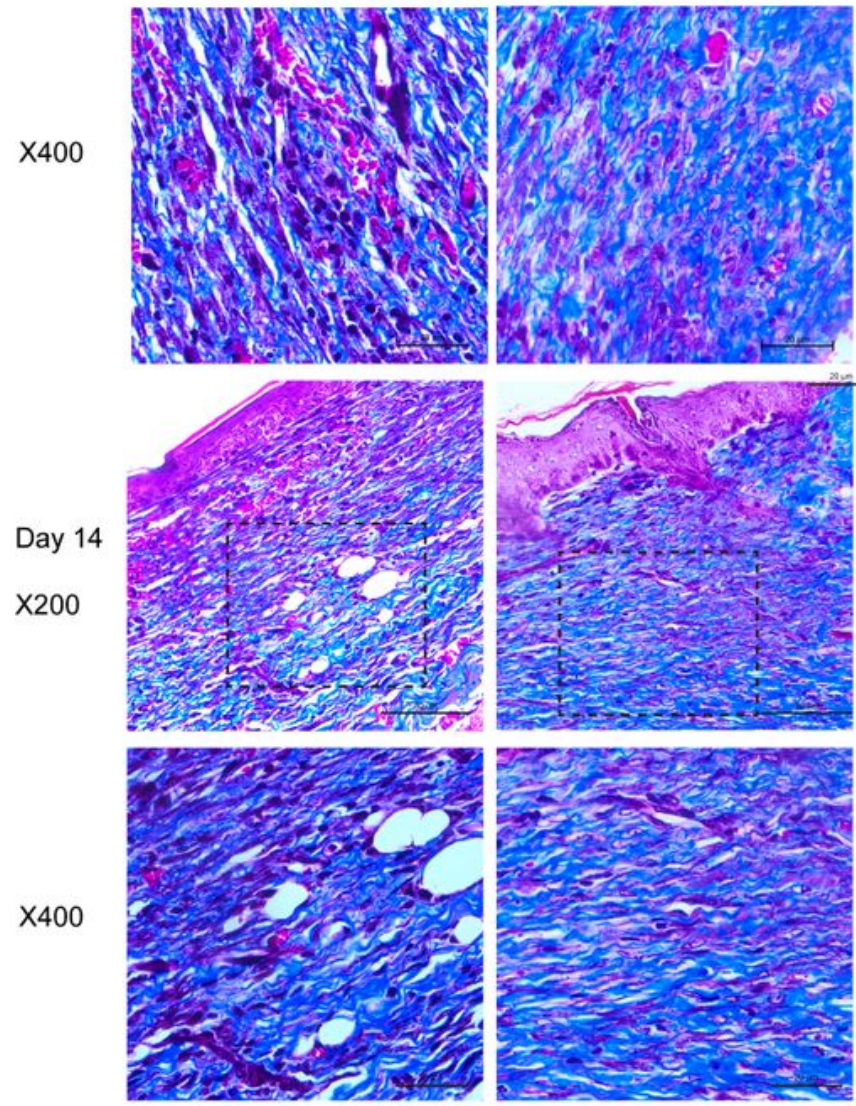

B

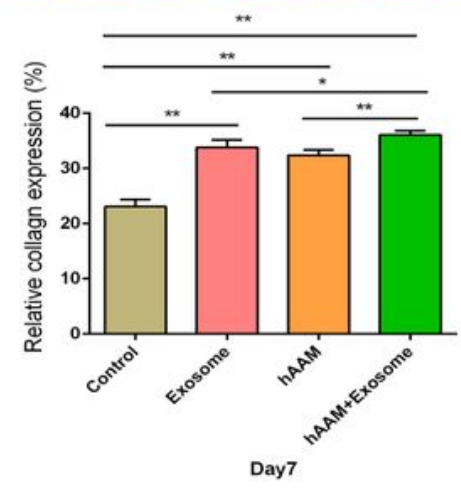

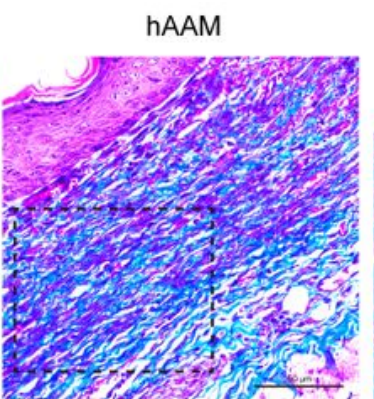
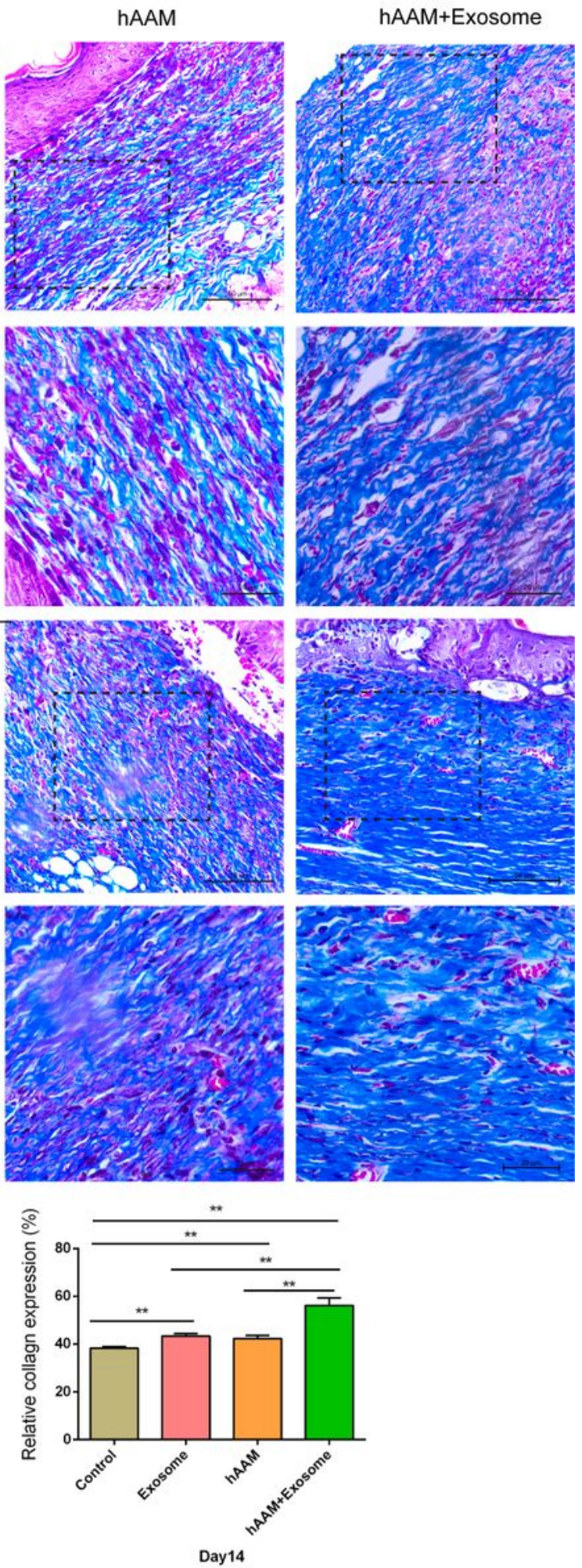

\section{Figure 7}

Regeneration of collagen was analyzed by Masson's trichrome staining. The hAAM-Exos group showed the highest amount of deposited collagen compared to the other three groups. (A) Representative images of collagen evaluated by H\&E staining in all groups at days 7 and 14 . The $200 \times$ image scale bar $=50 \mu \mathrm{m}$, the $400 \times$ image scale bar $=20 \mu \mathrm{m}$. (B) Quantitative analysis of the collagen regeneration in each group at day 7 and day 14 . ${ }^{* *} \mathrm{P}<0.01$, ${ }^{*} \mathrm{P}<0.05$. 
A

Control

Exosome

hAAM

hAAM+Exosome

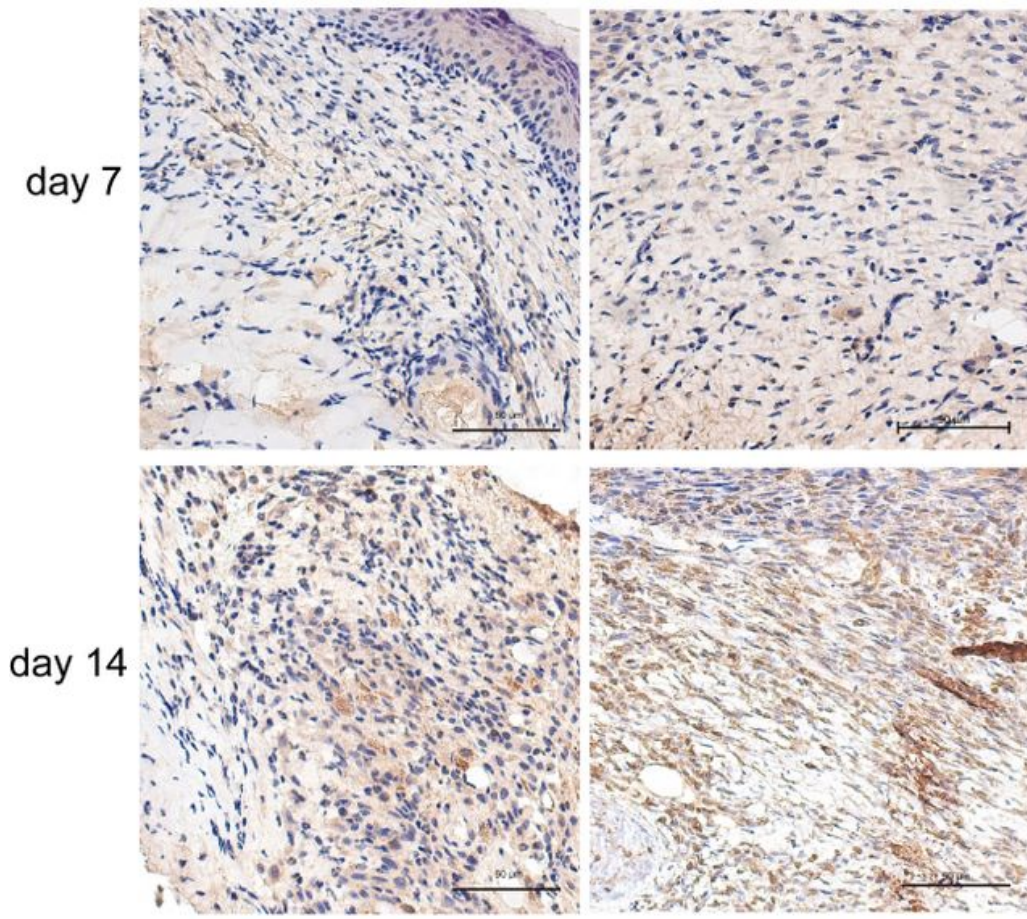

B

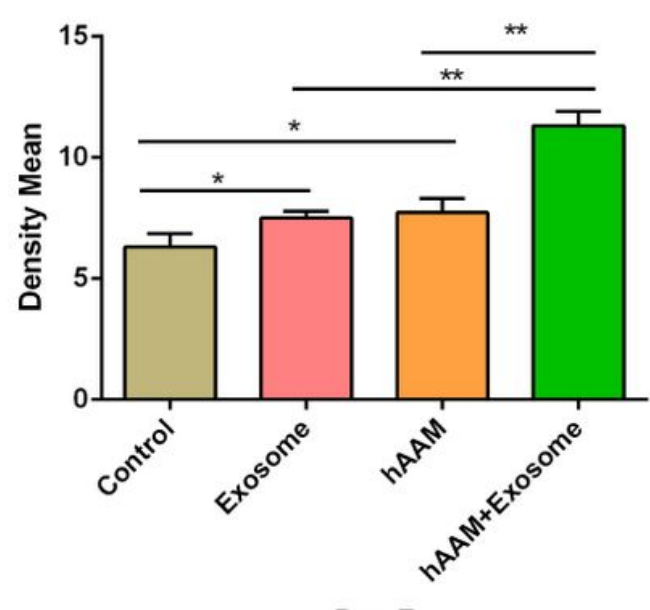

Day 7
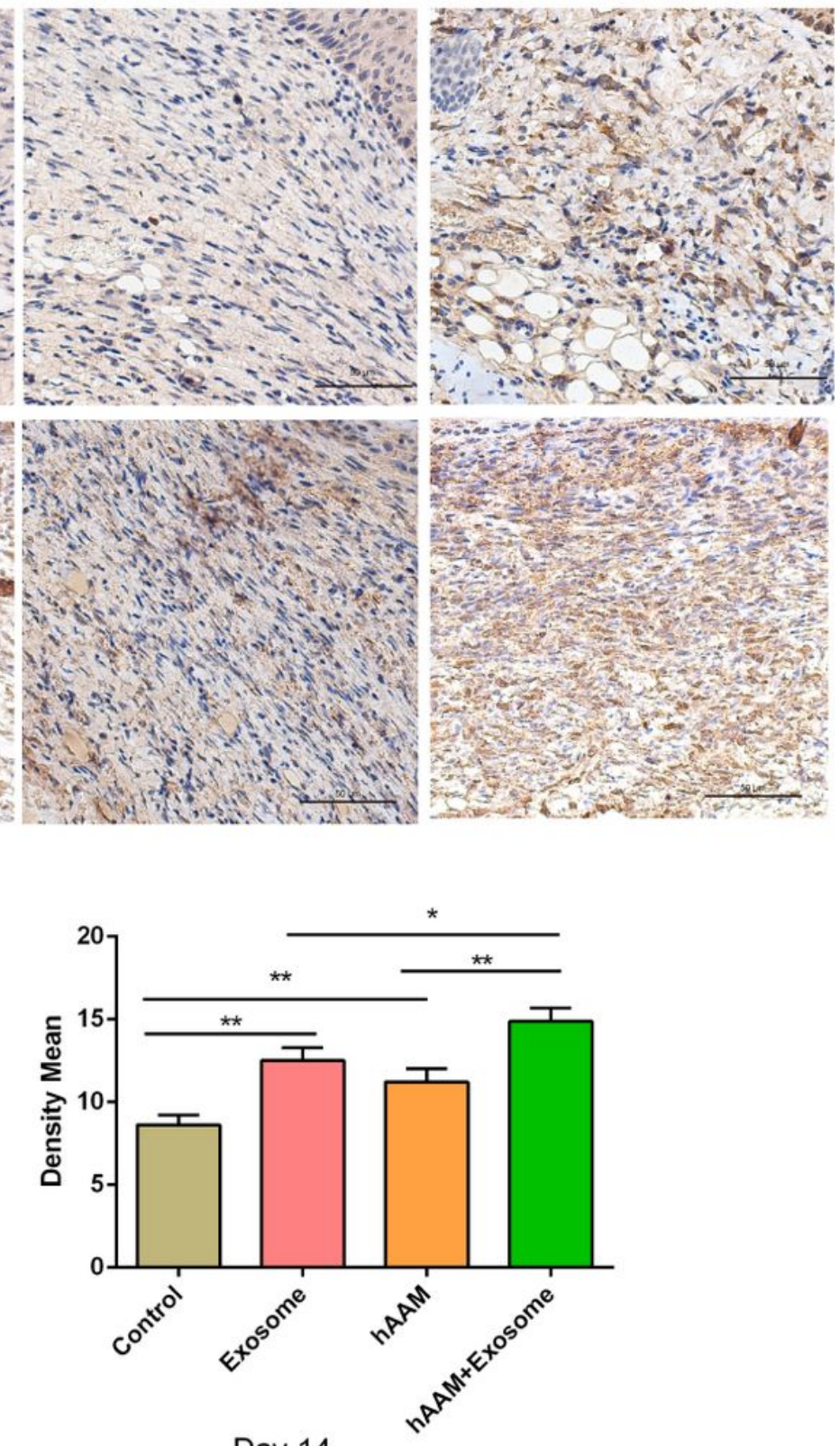

Day 14

Figure 8

Regeneration of collagen was analyzed by immunostaining of collagen type III. (A and B) Representative images and quantification results of collagen type III evaluated by immunohistochemical staining at days 7 and 14 . Scale bar $=50 \mu \mathrm{m} ;{ }^{* *} \mathrm{P}<0.01,{ }^{*} \mathrm{P}<0.05$.

\section{Supplementary Files}

This is a list of supplementary files associated with this preprint. Click to download.

- Schematic.jpg 